\title{
Grappes de multimorbidité chez les Autochtones et chez les non-Autochtones d'origine blanche au Canada
}

\author{
J. P. Kuwornu, M. Sc. (1); L. M. Lix, Ph. D. (1); S. Shooshtari, Ph. D. (1, 2)
}

Cet article a fait l'objet d'une évaluation par les pairs.

Diffuser cet article sur Twitter

\section{Résumé}

Introduction : Les profils de multimorbidité, c'est-à-dire la présence concomitante de deux maladies chroniques ou plus, ne sont pas nécessairement constants d'une population à l'autre. Les objectifs de notre étude étaient de comparer les estimations relatives à la prévalence de la multimorbidité entre une population canadienne autochtone et une population appariée non autochtone d'origine blanche et de définir les grappes de maladies chroniques caractérisant ces deux groupes.

Méthodologie : Nous avons utilisé les données de l'Enquête sur la santé dans les collectivités canadiennes (ESCC) pour cibler les répondants adultes (18 ans et plus) ayant déclaré être d'origine autochtone ou d'origine non autochtone blanche et être atteints d'au moins 2 des 15 maladies chroniques les plus fréquentes visées par l'ESCC. Les Autochtones répondant à ces critères ont été appariés en fonction du sexe et de l'âge à des non-Autochtones d'origine blanche. Les analyses ont été stratifiées par âge (18 à 54 ans, 55 ans et plus). Nous avons estimé la prévalence en utilisant la pondération employée dans l'enquête. Nous avons procédé à une analyse de structure latente (ASL) pour définir les grappes de maladies.

Résultats : Nous avons apparié 1642 répondants autochtones au même nombre de répondants non autochtones d'origine blanche. Sur l'ensemble, 38,9\% (IC à $95 \%$ : 36,5 \% à 41,3\%) des Autochtones étaient atteints de deux maladies chroniques ou plus, contre 30,7 \% (IC à $95 \%: 28,9 \%$ à 32,6 \%) des non-Autochtones. La comparaison des résultats de l'ASL a révélé que le meilleur ajustement aux données était obtenu avec trois grappes ou avec quatre grappes. Alors que les maladies concomitantes qui se manifestaient chez les personnes les plus âgées des deux populations avaient tendance à être similaires, les groupes plus jeunes de ces deux populations présentaient des différences.

Conclusion : Un petit groupe de répondants autochtones dans le groupe des plus jeunes présentait un profil complexe de maladies chroniques concomitantes. Ces personnes tireraient sans doute grand profit de programmes de gestion des maladies.

Mots-clés : Autochtone, maladie chronique, analyse de structure latente, prévalence

\section{Introduction}

La multimorbidité, c'est-à-dire la présence concomitante de plusieurs maladies chroniques dont l'une n'est pas plus centrale que l'autre ${ }^{1}$, est associée à une utilisation accrue des services de santé et à une qualité de vie moindre ${ }^{2-4}$. La multimorbidité se distingue ainsi de la comorbidité, caractérisée par la présence d'une maladie centrale (c.-à-d. primaire). La prévalence de la multimorbidité varie en fonction de facteurs de risque comme le sexe $^{1}$, l'âge ${ }^{2}$, le statut socioéconomique $\mathrm{e}^{5,6}$ et l'origine ethnique ${ }^{7}$. Les pays dont la population est diverse sur le plan socioculturel sont de ce fait susceptibles de rencontrer des difficultés spécifiques quant à la prestation de soins dans un contexte de multimorbidité ${ }^{8}$.

Identifier les maladies chroniques concomitantes peut contribuer à l'amélioration des stratégies de gestion des soins pour les patients à multimorbidité des groupes à risque élevé. Certains chercheurs ont fait état de la prévalence de combinaisons bien précises de maladies, alors que d'autres ont plutôt examiné les profils de multimorbidité en utilisant des techniques comme l'analyse en grappes $^{9-13}$. L'analyse de structure latente (ASL) est une technique permettant de définir des groupes de maladies apparentées (c.-à-d. des classes latentes). Cette technique a déjà été utilisée dans des études portant sur des maladies chroniques ${ }^{14}$, mais jamais, à notre connaissance, pour analyser les profils de multimorbidité de populations différentes. Elle est pourtant préférable dans ce cas aux méthodes classiques d'analyse en grappes car elle utilise des méthodes de classification fondées sur les probabilités et offre divers tests diagnostiques permettant de déterminer le nombre optimal de classes ${ }^{15}$.

Peu d'études ont porté sur la prévalence de maladies chroniques concomitantes au sein de groupes à risque différents dans une même population. Schafer et collab. ${ }^{9}$ ont comparé des grappes de maladies chroniques chez des aînés, hommes et femmes, en Allemagne. Ils ont décelé trois grappes de maladies dans les deux groupes, mais ont constaté des différences quant aux maladies chroniques en cause

Rattachement des auteurs :

1. Département des sciences de la santé communautaire, Faculté de médecine, Université du Manitoba, Winnipeg (Manitoba), Canada 2. Département des sciences sociales de la famille, Université du Manitoba, Winnipeg (Manitoba), Canada

Correspondance : John Paul Kuwornu, Département des sciences de la santé communautaire, Faculté de médecine, Université du Manitoba, Winnipeg (Manitoba) R3E 0W3; tél. : 204-789-3573; téléc. : 204-789-3905; courriel : kuwornuj@myumanitoba.ca 
dans chacune des grappes. Par exemple, les femmes de l'une des grappes étaient relativement plus souvent atteintes d'une affection préterminale comme une cardiopathie ischémique chronique ou une insuffisance rénale.

Comme la prévalence des maladies chroniques est à la hausse chez les Autochtones, c'est-à-dire chez les Premières nations, les Métis et les Inuits ${ }^{16}$, la possibilité que la fréquence des cas de multimorbidité soit à la hausse est particulièrement préoccupante. Cependant, aucune étude n'a examiné les taux de prévalence au sein de cette population, ou n'a cherché à déterminer si les maladies chroniques ayant tendance à être présentes en grappe étaient différentes chez les Autochtones et les nonAutochtones. Par exemple, la prévalence du diabète, qui augmente plus rapidement chez les Autochtones que chez les nonAutochtones au Canada ${ }^{17}$, pourrait provoquer des grappes de maladies concomitantes connexes, comme les maladies rénales et les maladies cardiovasculaires.

Nos objectifs étaient d'estimer la prévalence de la multimorbidité chez les Autochtones du Canada et de comparer cette prévalence aux estimations correspondant à une population appariée non autochtone d'origine blanche, puis de comparer les grappes de maladies chroniques concomitantes observées chez ces deux populations en fonction de deux groupes d'âge.

\section{Méthodologie}

\section{Populations et variables de l'étude}

Les données que nous avons utilisées sont tirées du cycle 3.1 de l'Enquête sur la santé dans les collectivités canadiennes (ESCC) de Statistique Canada ${ }^{18}$. L'ESCC est une étude transversale en population qui vise à fournir de l'information sur des déterminants de la santé, sur l'état de santé et sur l'utilisation des services de soins de santé dans 122 régions sanitaires du Canada. L'enquête a été menée tous les 2 ans de 2001 à 2005 et elle est annuelle depuis 2007. Nous avons choisi le cycle 3.1 plutôt que le cycle 4.1, car le nombre de répondants autochtones y est suffisamment important pour que nous puissions effectuer des ASL pour le groupe des plus jeunes et pour le groupe des plus âgés. La population ciblée dans le cycle 3.1 de l'ESCC est celle des individus de 12 ans ou plus vivant dans un logement privé dans une province ou un territoire du Canada. L'ESCC fait appel à une méthode d'échantillonnage en grappes stratifiée à plusieurs degrés pour cibler les personnes admissibles et les membres de leur ménage $(n=132$ 221; taux de réponse = 92,9\%). Sont exclus de l'ESCC les personnes hébergées en institution, les membres des Premières nations vivant dans une réserve et les membres à plein temps des Forces canadiennes.

Les critères d'inclusion pour notre étude étaient : 1) avoir au moins 18 ans (de façon à cibler la population d'âge adulte), 2) s'être autodéfini soit comme Autochtone, soit comme non-Autochtone d'origine blanche et 3) avoir déclaré être atteint d'au moins 2 des 15 maladies chroniques les plus fréquentes visées par le cycle 3.1 de l'ESCC. Nous avons sélectionné la population autochtone en nous fondant sur la variable calculée fournie dans le cycle 3.1 de l'ESCC, qui combine l'information fournie en réponse à deux variantes d'une question. Avant juin 2005, la question avait en préambule : " Les gens qui habitent au Canada ont des origines culturelles et raciales très variées. " Ce préambule était suivi de la question elle-même : « Êtes-vous un Autochtone (Indien de l'Amérique du Nord, Métis, Inuit)? » Nous avons considéré les répondants comme étant autochtones s'ils avaient répondu " oui » à cette question. En juin 2005, la question a été modifiée ainsi : " Êtes-vous un Autochtone, c'est-à-dire un Indien de l'Amérique du Nord, un Métis ou un Inuit? » La population non autochtone d'origine blanche a été sélectionnée à l'aide d'une variable calculée similaire fournie dans le cycle 3.1 de l'ESCC $^{19}$.

Nous avons effectué un appariement de type "un à un » entre les répondants autochtones et aux non autochtones d'origine blanche qui répondaient aux critères d'inclusion de notre étude en utilisant comme critères le sexe et l'âge (par tranche de 5 ans). Nous avons pu apparier chaque répondant autochtone à un répondant non autochtone d'origine blanche. Lorsqu'il y avait plus d'un répondant non autochtone d'origine blanche pouvant être apparié à un répondant autochtone, nous avons procédé à un appariement aléatoire. Un appariement de type " un à plusieurs » aurait été avantageux si la prévalence d'une ou de plusieurs maladies chroniques avait été considérablement moins élevée chez les non-Autochtones d'origine blanche que chez les Autochtones, ce qui aurait assuré un degré de précision suffisant en ce qui concerne les estimations de la prévalence, mais il n'y avait aucune différence notable à cet égard entre les deux populations à l'étude. Bien que le fait d'éliminer des individus lors du processus d'appariement de type " un à un » a pour conséquence de réduire la taille de l'échantillon, cela n'entraîne pas nécessairement une augmentation de la variance d'échantillonnage pour les estimations. De façon générale, l'appariement améliore l'équilibre dans la distribution des covariables, ce qui peut diminuer la variance associée aux estimateurs ${ }^{20}$. L'appariement a été effectué afin que les populations soient comparables pour deux variables démographiques dont on sait qu'elles sont associées à des maladies chroniques (l'âge et le sexe). Nous avons ensuite divisé les répondants en deux groupes, un groupe plus jeune (18 à 54 ans) et un groupe plus âgé (55 ans ou plus) afin d'étudier les différences éventuelles entre les grappes de maladies observées au sein du groupe des répondants les plus jeunes et au sein du groupe des répondants les plus âgés de chaque population.

N'ont été retenues ici pour l'analyse en grappes que les maladies associées à un taux de prévalence d'au moins $5 \%$, à la fois chez les Autochtones et chez les nonAutochtones d'origine blanche appariés, en fonction des estimations pondérées de la prévalence. Le préambule aux questions de l'ESCC portant sur les maladies chroniques était : « Les questions qui suivent portent sur certains problèmes de santé de longue durée que vous pouvez avoir. Par "problème de santé de longue durée", on entend un état qui dure ou qui devrait durer 6 mois ou plus et qui a été diagnostiqué par un professionnel de la santé. » Les répondants devaient ensuite indiquer s'ils souffraient ou non de chacune des maladies mentionnées par l'enquêteur ${ }^{19}$. Nous avons codé les 
réponses portant sur chacune des maladies en « oui » ou « non ». Toutes les autres réponses (c'est-à-dire "ne sait pas » ou refus de réponse) ont été traitées comme des valeurs manquantes. Les 15 maladies chroniques visées par notre étude étaient l'asthme, l'arthrite ou les rhumatismes, l'anxiété ou les troubles mentaux, les maux de dos, les troubles intestinaux, la cataracte, le diabète, l'emphysème ou la bronchite ou une maladie pulmonaire obstructive chronique (MPOC), les allergies alimentaires, les maladies cardiaques, l'hypertension, l'incontinence, les migraines, un problème de thyroïde et les ulcères. La multimorbidité a été définie par la présence d'au moins deux de ces maladies.

\section{Analyse statistique}

Nous avons décrit les groupes de répondants autochtones et de répondants non autochtones d'origine blanche correspondants selon l'âge, le sexe et la prévalence de chacune des 15 maladies chroniques citées ci-dessus. La prévalence de la multimorbidité a été estimée avec des intervalles de confiance (IC) à $95 \%$. Dans le cas de la population autochtone, le numérateur de la formule de la prévalence était le nombre de répondants autochtones qui répondaient aux critères d'inclusion de l'étude, tandis que le dénominateur était le nombre total de répondants autochtones de 18 ans ou plus. Pour ce qui est de la population non autochtone d'origine blanche, le numérateur était le nombre de répondants non autochtones d'origine blanche appariés répondant aux critères d'inclusion de l'étude, tandis que le dénominateur était le nombre total de répondants non autochtones d'origine blanche âgés de 18 ans ou plus répondant aux critères d'appariement. Les estimations de la prévalence sont exprimées en pourcentages.

Nous avons appliqué l'ASL séparement aux groupes autochtones plus jeunes et plus âgés et aux aux répondants non autochtones d'origine blanche qui leur ont été appariés. L'ASL suppose que chaque personne participant à l'étude appartient à l'une ou l'autre d'un ensemble de classes s'excluant mutuellement et exhaustives ${ }^{21}$. Nous avons calculé les probabilités d'appartenance à une classe, c'est-à-dire les estimations de la proportion de répondants appartenant à chaque classe latente, ainsi que les probabilités de réponse à un élément (présence d'une maladie) en fonction de l'appartenance à une classe, c'est-àdire les estimations de prévalence des maladies chroniques pour chacune des classes latentes ${ }^{21}$. La probabilité de réponse à un élément sert à caractériser les classes latentes de la même manière que les saturations servent à caractériser les facteurs dans l'analyse factorielle. Selon notre interprétation, une probabilité de réponse à un élément de 0,4 ou plus indique une association entre l'élément et la classe latente correspondante, ce qui concorde avec les études antérieures sur la multimorbidité fondées sur l'analyse factorielle, où l'on définit une valeur minimale de 0,4 pour les saturations de facteurs ${ }^{22}$.

Pour déterminer le nombre optimal de classes, nous avons ajusté les modèles aux données en utilisant entre deux et sept classes. Nous nous sommes servis du critère d'information d'Akaike (AIC) ${ }^{23}$ et du critère bayésien de Schwarz $(\mathrm{SBC})^{24}$, qui sont des mesures de pénalisation de la fonction de vraisemblance, pour déterminer le nombre définitif de classes ${ }^{25}$ (figure 1). Selon le principe d'arbitrage entre l'ajuste-

\section{FIGURE 1}

Valeurs du critère d'information d'Akaike et du critère bayésien de Schwarz pour les modèles d'analyse de structure latente chez les répondants autochtones et les répondants non autochtones d'origine blanche appariés de l'ESCC

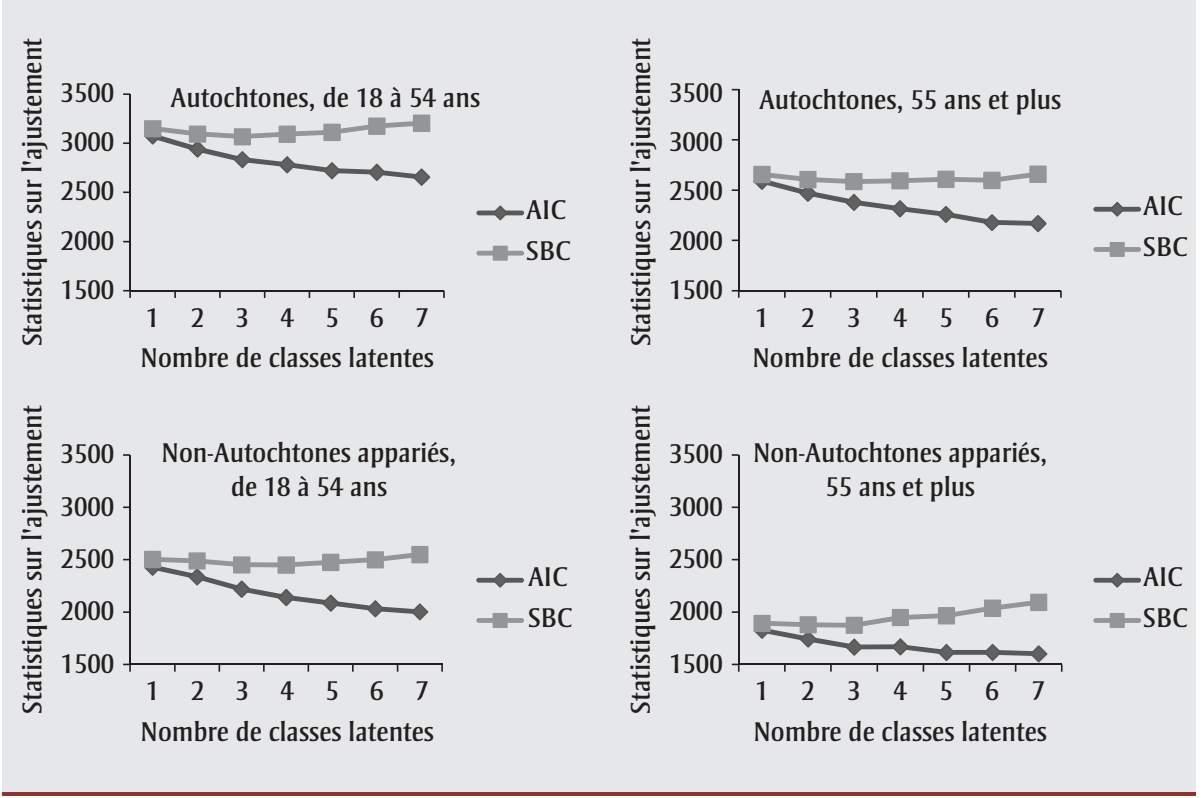

Abréviations : AIC, critère d'information d'Akaike; ESCC, Enquête sur la santé dans les collectivités canadiennes; SBC, critère bayésien de Schwarz. ment et la parcimonie, on a choisi le modèle pour lequel les valeurs de l'AIC et du SBC étaient relativement peu élevées.

Nous avons aussi testé l'invariance des mesures entre les répondants autochtones et les répondants non autochtones appariés. Pour ce faire, nous avons tout d'abord ajusté un modèle où les paramètres des réponses aux éléments ont été librement estimés pour les deux groupes. Nous avons ensuite ajusté un second modèle aux données dans lequel les paramètres ont été fixés identiquement entre les groupes. La différence entre les statistiques du rapport de vraisemblance des deux modèles imbriqués, $G^{2}$, est distribuée asymptotiquement selon la loi du $\chi^{2}$, le nombre de degrés de liberté $(d l)$ étant égal à la différence du nombre de degrés de liberté entre les deux modèles imbriqués. Si l'hypothèse nulle de l'invariance des mesures n'est pas rejetée, on présume alors que les classes définies sont les mêmes pour les deux groupes. Si l'hypothèse nulle est rejetée, il est recommandé d'établir des estimations distinctes pour des classes des deux groupes ${ }^{21}$. Nous avons effectué des tests distincts pour les populations plus jeunes et pour les plus âgées. Dans chaque cas, seuls les modèles 
optimaux obtenus en fonction des statistiques d'ajustement et des résultats concernant l'interprétabilité du modèle ont servi à tester l'invariance des mesures.

L'analyse a été réalisée au moyen de la version PROC LCA $1.3 .0^{26}$ du logiciel SAS, version $9.3^{27}$. Tous les calculs ont été effectués au moyen des poids de l'échantillon intégral. L'autorisation d'accéder aux données a été accordée par Statistique Canada.

\section{Résultats}

Les 1642 répondants autochtones (n pondéré : 198 955) qui répondaient aux critère d'inclusion de l'étude ont été appariés à un nombre égal de répondants non autochtones d'origine blanche (n pondéré : 169 149). Le tableau 1 présente les caractéristiques des Autochtones et des non-Autochtones d'origine blanche appariés, en fonction des deux groupes d'âge. L'âge moyen de la cohorte d'Autochtones plus jeunes (18 à 54 ans) était de 37,4 ans (écart-type : 17,8 ans), et $39,4 \%$ des répondants étaient de sexe masculin. L'âge moyen de la cohorte d'Autochtones plus âgés (55 ans et plus) était de 64,8 ans (écart-type : 10,8 ans) et 41,9 \% des répondants étaient de sexe masculin. La distribution des répondants en fonction de l'âge et du sexe était semblable entre les non-Autochtones et les Autochtones étant donné le processus d'appariement choisi.
Le nombre de maladies chroniques était plus élevé chez les Autochtones que chez les non-Autochtones, tant dans le groupe des plus jeunes que dans le groupe des plus âgés. Les maux de dos étaient le problème de santé le plus fréquent chez les jeunes, qu'ils soient autochtones (55,8 \%) ou non autochtones $(50,8 \%)$. Chez les plus âgés, l'arthrite ou les rhumatismes étaient la maladie la plus fréquente chez les Autochtones $(63,4 \%)$ comme chez les non-autochtones $(64,5 \%)$.

Globalement, la prévalence de la multimorbidité était de 38,9 \% (IC à $95 \%$ : 36,5 \% à $41,3 \%$ ) chez les Autochtones et de $30,7 \%$ (IC à $95 \%: 28,9 \%$ à $32,6 \%$ ) chez les non-Autochtones. La prévalence de la

TABLEAU 1

Caractéristiques démographiques et maladies chroniques des répondants autochtones et des répondants appariés non autochtones d'origine blanche de I'ESCC, par groupe d'âge

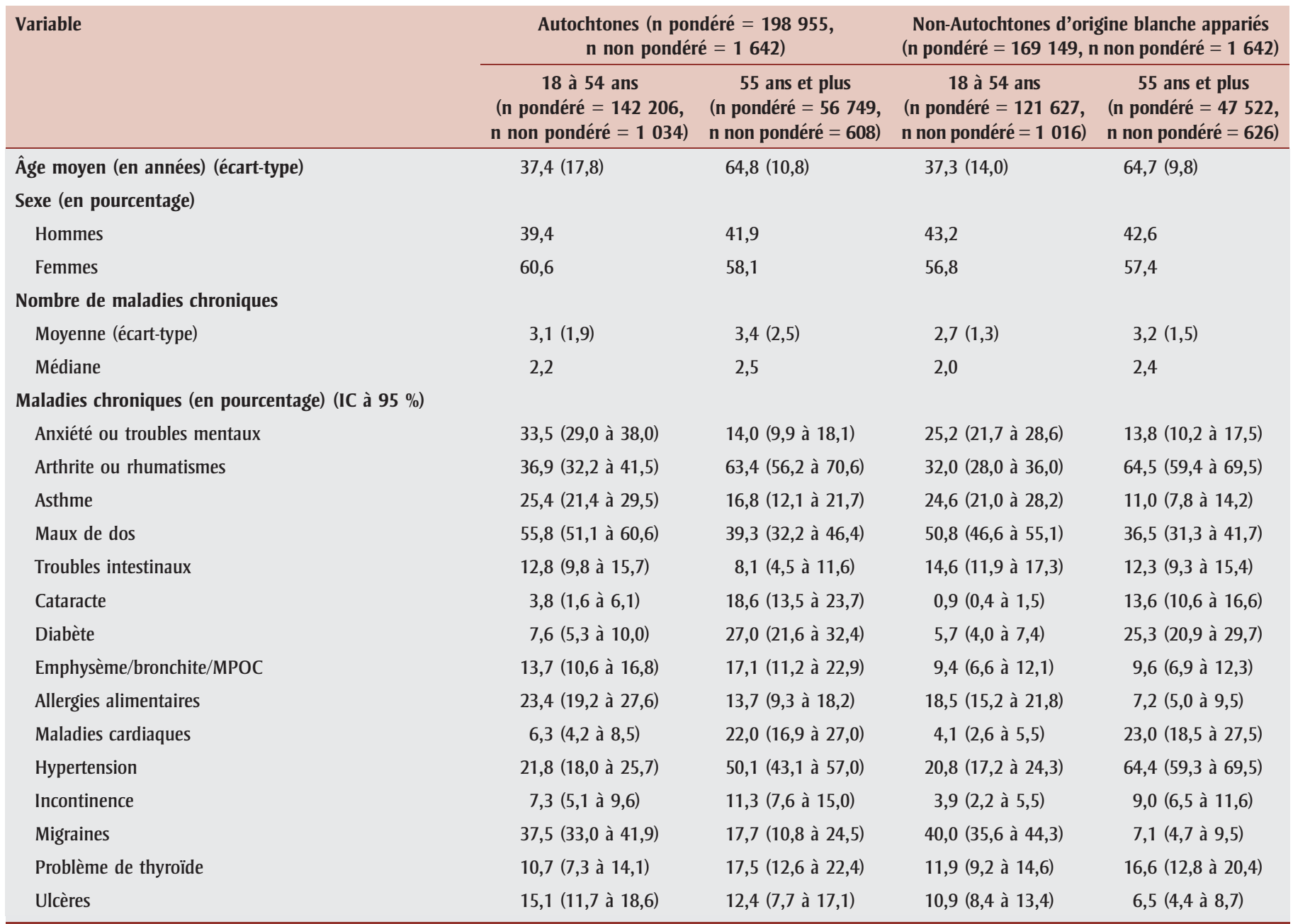

Abréviations : ESCC, Enquête sur la santé dans les collectivités canadiennes; MPOC, maladie pulmonaire obstructive chronique.

Remarque : Il existe de légères différences dans la distribution en fonction du sexe et de l'âge entre les Autochtones et les non-Autochtones d'origine blanche appariés en raison de la pondération utilisée dans l'enquête. 
multimorbidité était plus élevée chez les Autochtones plus âgés $(64,0 \%$; IC à $95 \%$ : $58,7 \%$ à $69,2 \%$ ) que chez les nonAutochtones plus âgés (58,3\% ; IC à $95 \%$ : $53,7 \%$ à 63,0 \% ). De même, la prévalence de la multimorbidité était plus élevée chez les Autochtones plus jeunes (33,7\%; IC à $95 \%: 31,1 \%$ à 36,2\%) que chez les nonAutochtones plus jeunes (25,7 \%; IC à $95 \%$ : 23,6 \% à 27,7 \%) (données non présentées).

Les résultats de l'ASL sont présentés sur la figure 1. Alors que les valeurs de l'AIC diminuent avec l'augmentation du nombre de classes latentes dans les deux populations et pour les deux groupes d'âge, le SCB a plutôt augmenté (signe d'un moins bon ajustement) après trois classes dans les deux groupes d'âge chez les Autochtones, et dans le groupe de sujets plus âgés chez les non-Autochtones. Les valeurs du SBC ont commencé à augmenter après quatre classes dans le groupe de non-Autochtones plus jeunes. Par conséquent, nous avons choisi de comparer le modèle à trois classes et le modèle à quatre classes pour les deux groupes.

L'invariance des mesures entre les Autochtones et les non-Autochtones était associée, chez les répondants plus jeunes, à une valeur $G^{2}$ de 192,6 $(d l=45)$ pour le modèle à 3 classes $(p<0,0001)$ et de $224,0(d l=60)$ pour le modèle à 4 classes ( $p<0,0001)$. Chez les répondants plus âgés, l'invariance des mesures entre les Autochtones et les non-Autochtones a entraîné une valeur $G^{2}$ de 189,6 $(d l=45)$ pour le modèle à 3 classes $(p<0,0001)$ et de $182,3(d l=60)$ pour le modèle à 4 classes $(p<0,0001)$. Ces résultats laissent penser qu'il existe une non-invariance des mesures dans les données et, par conséquent, nous avons estimé séparément les paramètres du modèle d'ASL pour les répondants autochtones et pour les répondants non autochtones dans chacun des groupes d'âge.

\section{Caractéristiques du modèle à trois classes}

Le tableau 2 montre le pourcentage d'appartenance à chacune des classes et les probabilités de réponses à un élément pour trois classes. Chez les plus jeunes, la

TABLEAU 2

Probabilité de réponse à un élément pour plusieurs maladies chroniques, répondants autochtones et répondants non autochtones d'origine blanche appariés de l'ESCC, par groupe d'âge, modèle à trois classes

\begin{tabular}{|c|c|c|c|c|c|c|}
\hline \multirow[t]{3}{*}{ Maladies chroniques } & \multicolumn{6}{|c|}{18 à 54 ans } \\
\hline & \multicolumn{3}{|c|}{ Autochtones } & \multicolumn{3}{|c|}{$\begin{array}{l}\text { Non-Autochtones d'origine } \\
\text { blanche appariés }\end{array}$} \\
\hline & $\begin{array}{l}\text { Classe } 1 \\
(5,8 \%)\end{array}$ & $\begin{array}{l}\text { Classe } 2 \\
(18,3 \%)\end{array}$ & $\begin{array}{l}\text { Classe } 3 \\
(75,9 \%)\end{array}$ & $\begin{array}{l}\text { Classe } 1 \\
(11,9 \%)\end{array}$ & $\begin{array}{l}\text { Classe } 2 \\
(27,6 \%)\end{array}$ & $\begin{array}{l}\text { Classe } 3 \\
(60,5 \%)\end{array}$ \\
\hline Anxiété ou troubles mentaux & 0,678 & 0,189 & 0,344 & 0,230 & 0,168 & 0,294 \\
\hline Arthrite ou rhumatismes & 0,632 & 0,312 & 0,362 & 0,158 & 0,292 & 0,364 \\
\hline Asthme & 0,510 & 0,063 & 0,281 & 1,000 & 0,183 & 0,127 \\
\hline Maux de dos & 0,600 & 0,376 & 0,599 & 0,267 & 0,356 & 0,626 \\
\hline Troubles intestinaux & 0,189 & 0,080 & 0,135 & 0,144 & 0,062 & 0,184 \\
\hline Cataracte & 0,256 & 0,000 & 0,031 & 0,003 & 0,015 & 0,008 \\
\hline Diabète & 0,244 & 0,158 & 0,045 & 0,023 & 0,135 & 0,028 \\
\hline Emphysème/bronchite/MPOC & 0,541 & 0,008 & 0,138 & 0,203 & 0,058 & 0,089 \\
\hline Allergies alimentaires & 0,181 & 0,167 & 0,254 & 0,356 & 0,096 & 0,192 \\
\hline Maladies cardiaques & 0,436 & 0,080 & 0,031 & 0,000 & 0,128 & 0,009 \\
\hline Hypertension & 0,645 & 0,989 & 0,000 & 0,000 & 0,751 & 0,000 \\
\hline Incontinence & 0,268 & 0,036 & 0,068 & 0,000 & 0,024 & 0,053 \\
\hline Migraines & 0,608 & 0,248 & 0,387 & 0,055 & 0,290 & 0,518 \\
\hline Problème de thyroïde & 0,116 & 0,070 & 0,115 & 0,050 & 0,110 & 0,137 \\
\hline Ulcères & 0,515 & 0,078 & 0,142 & 0,053 & 0,124 & 0,114 \\
\hline \multirow[t]{3}{*}{ Maladies chroniques } & \multicolumn{6}{|c|}{55 ans et plus } \\
\hline & \multicolumn{3}{|c|}{ Autochtones } & \multicolumn{3}{|c|}{$\begin{array}{l}\text { Non-Autochtones d'origine } \\
\text { blanche appariés }\end{array}$} \\
\hline & $\begin{array}{l}\text { Classe } 1 \\
(14,7 \%)\end{array}$ & $\begin{array}{l}\text { Classe } 2 \\
(52,7 \%)\end{array}$ & $\begin{array}{l}\text { Classe } 3 \\
(32,6 \%)\end{array}$ & $\begin{array}{l}\text { Classe } 1 \\
(31,4 \%)\end{array}$ & $\begin{array}{l}\text { Classe } 2 \\
(39,8 \%)\end{array}$ & $\begin{array}{l}\text { Classe } 3 \\
(28,8 \%)\end{array}$ \\
\hline Anxiété ou troubles mentaux & 0,328 & 0,084 & 0,146 & 0,126 & 0,082 & 0,230 \\
\hline Arthrite ou rhumatismes & 0,931 & 0,591 & 0,575 & 0,734 & 0,546 & 0,683 \\
\hline Asthme & 0,780 & 0,056 & 0,076 & 0,188 & 0,095 & 0,045 \\
\hline Maux de dos & 0,608 & 0,233 & 0,555 & 0,310 & 0,212 & 0,636 \\
\hline Troubles intestinaux & 0,190 & 0,025 & 0,122 & 0,225 & 0,000 & 0,183 \\
\hline Cataracte & 0,121 & 0,194 & 0,202 & 0,290 & 0,113 & 0,000 \\
\hline Diabète & 0,398 & 0,401 & 0,000 & 0,256 & 0,434 & 0,000 \\
\hline Emphysème/bronchite/MPOC & 0,508 & 0,085 & 0,157 & 0,307 & 0,000 & 0,000 \\
\hline Allergies alimentaires & 0,156 & 0,102 & 0,186 & 0,103 & 0,022 & 0,109 \\
\hline Maladies cardiaques & 0,246 & 0,297 & 0,084 & 0,353 & 0,223 & 0,105 \\
\hline Hypertension & 0,549 & 0,751 & 0,076 & 0,539 & 0,886 & 0,423 \\
\hline Incontinence & 0,200 & 0,085 & 0,120 & 0,212 & 0,023 & 0,051 \\
\hline Migraines & 0,306 & 0,081 & 0,273 & 0,081 & 0,024 & 0,126 \\
\hline Problème de thyroïde & 0,284 & 0,155 & 0,160 & 0,144 & 0,088 & 0,297 \\
\hline Ulcères & 0,187 & 0,031 & 0,246 & 0,067 & 0,047 & 0,088 \\
\hline
\end{tabular}

Abréviations : ESCC, Enquête sur la santé dans les collectivités canadiennes; MPOC, maladie pulmonaire obstructive chronique. Remarque : Les valeurs en gras indiquent les maladies pour lesquelles la probabilité de réponse était de 0,400 ou plus.

première classe latente comptait moins de $12 \%$ des répondants des deux populations (5,8\% chez les Autochtones et $11,9 \%$ chez les non-Autochtones). Toujours chez les plus jeunes, la deuxième classe comptait près du cinquième $(18,3 \%)$ des répondants chez les Autochtones et plus du quart $(27,6 \%)$ des répondants chez les non-Autochtones. Chez les plus âgés, plus de la moitié $(52,7 \%)$ des répondants 
autochtones appartenaient à la deuxième classe contre 39,8 \% des répondants non autochtones.

\section{Groupe des plus jeunes}

En ce qui concerne le modèle à trois classes chez les Autochtones plus jeunes, la première classe était associée à une probabilité de réponse élevée pour huit des maladies chroniques, alors que la deuxième classe était associée à une probabilité de réponse élevée pour l'hypertension et que la troisième classe était associée à une probabilité de réponse élevée pour les maux de dos. Chez les non-Autochtones plus jeunes, les probabilités de réponse étaient élevées pour l'asthme dans la première classe, pour l'hypertension dans la deuxième classe et pour les maux de dos et les migraines dans la troisième classe.

\section{Groupe des plus âgés}

En ce qui concerne le modèle à trois classes chez les Autochtones plus âgés, les probabilités de réponse étaient élevées pour cinq maladies chroniques dans la première classe, pour l'arthrite ou les rhumatismes, le diabète et l'hypertension dans la deuxième classe et pour l'arthrite ou les rhumatismes et les maux de dos dans la troisième classe. Chez les nonAutochtones plus âgés, les probabilités de réponse les plus élevées concernaient l'arthrite ou les rhumatismes et l'hypertension dans la première classe. Dans la deuxième classe, il s'agissait des mêmes maladies que chez les répondants autochtones du même groupe d'âge (arthrite ou rhumatisme, diabète et hypertension). Dans la troisième classe, on retrouvait l'arthrite ou les rhumatismes, les maux de dos et l'hypertension.

Globalement, les résultats du modèle d'ASL à 3 classes révèlent qu'il est plus susceptible d'y avoir des grappes de maladies chroniques chez les sujets plus âgés, qu'ils soient autochtones ou non autochtones, que chez les sujets plus jeunes des deux populations.

\section{Caractéristiques du modèle à quatre classes}

\section{Groupe des plus jeunes}

Les différences observées entre les Autochtones et les non-Autochtones pour le groupe des plus jeunes dans le modèle à trois classes persistaient dans le modèle à quatre classes (tableau 3). Chez les Autochtones, la première classe latente est demeurée petite $(6,0 \%)$ et la probabilité de réponse était élevée pour les mêmes maladies. La deuxième classe comprenait le cinquième des répondants $(21 \%)$ et était associée à une probabilité élevée de réponse pour l'hypertension. La troisième classe, qui comprenait près des deux tiers $(62,4 \%)$ des répondants, était associée à une probabilité élevée de réponse pour les maux de dos. Dans la quatrième classe, la probabilité de réponse était élevée pour les maux de dos et les ulcères. Chez les répondants non autochtones, la première classe était également de petite taille $(13,1 \%)$, et elle était associée à une probabilité de réponse élevée pour l'asthme. La deuxième classe était associée à une probabilité élevée de réponse pour l'hypertension et la troisième classe, pour les migraines.

\section{Groupe des plus âgés}

En ce qui concerne le modèle à quatre classes chez les Autochtones plus âgés (tableau 3), la première classe comprenait près du cinquième de l'échantillon (17,6 \%) et était associée à une probabilité de réponse élevée pour 5 des 15 maladies chroniques. Dans la deuxième classe, la probabilité de réponse était élevée pour l'arthrite ou les rhumatismes et pour l'hypertension et, dans la troisième classe, pour l'arthrite ou les rhumatismes et les maux de dos. Dans la quatrième classe, qui comprenait la plus faible proportion de membres $(3,9 \%)$, la probabilité de réponse était élevée pour quatre maladies chroniques (maux de dos, cataracte, emphysème et ulcères). Chez les non-Autochtones plus âgés, la première classe $(9,3 \%)$ était associée à une probabilité de réponse élevée pour sept maladies chroniques. Le nombre de maladies associées à une probabilité de réponse élevée était moins important dans les autres classes. Il existait toutefois des similitudes quant aux grappes de maladies chez les Autochtones et les non-Autochtones.

\section{Analyse}

Il s’agit de la première étude représentative à l'échelle nationale à comparer la prévalence de la multimorbidité et à examiner les profils de maladies chroniques conco- mitantes chez une population autochtone et une population appariée non autochtone d'origine blanche. Nos résultats révèlent, chez les Autochtones, une prévalence de la multimorbidité plus élevée, à la fois globalement et pour la majorité des maladies chroniques étudiées.

Nous avons identifié, chez les répondants autochtones plus jeunes, un petit groupe dans le modèle à trois classes $(5,8 \%)$ et le modèle à quatre classes $(6,0 \%)$ chez qui la prévalence de maladies chroniques multiples était élevée. Nous n'avons pas observé de grappe présentant des caractéristiques similaires chez les répondants non autochtones d'origine blanche plus jeunes. Chez les sujets plus jeunes des deux populations, il n'y avait dans les autres grappes de maladies observées qu'une ou deux maladies qui étaient associées à une prévalence élevée dans le modèle à trois ou à quatre classes.

Dans le groupe des plus âgés, et à la différence du groupe des plus jeunes, les grappes de maladies chroniques comportaient fréquemment, dans les modèles à trois classes comme ceux à quatre classes, une série de trois maladies ou plus, et il y avait certaines similitudes entre les classes latentes identifiées chez les Autochtones et chez les non-Autochtones. Par exemple, dans le modèle à trois classes, l'arthrite ou les rhumatismes, le diabète et l'hypertension étaient associés dans les deux populations à la probabilité de concomitance la plus élevée.

Certaines des grappes de maladies chroniques identifiées dans notre étude ont déjà été décelées dans le cadre de travaux antérieurs. Une étude menée sur un échantillon d'Australiens en âge de travailler a révélé l'existence de six grappes de maladies chroniques ${ }^{22}$. Ces chercheurs ont constaté que l'arthrite, les maux de dos ou de cou, les migraines et une série d'autres maladies associées à de la douleur chronique avaient tendance à être concomitants. Ces résultats sont similaires aux nôtres, en ce sens que nous avons aussi constaté que l'arthrite ou les rhumatismes et les maux de dos avaient tendance à être concomitants. Nous avons également décelé une grappe de maladies, chez les sujets non autochtones d'origine blanche plus âgés, 
TABLEAU 3

Probabilité de réponse à un élément pour plusieurs maladies chroniques, répondants autochtones et répondants non autochtones d'origine blanche appariés de l'ESCC, par groupe d'âge, modèle à quatre classes

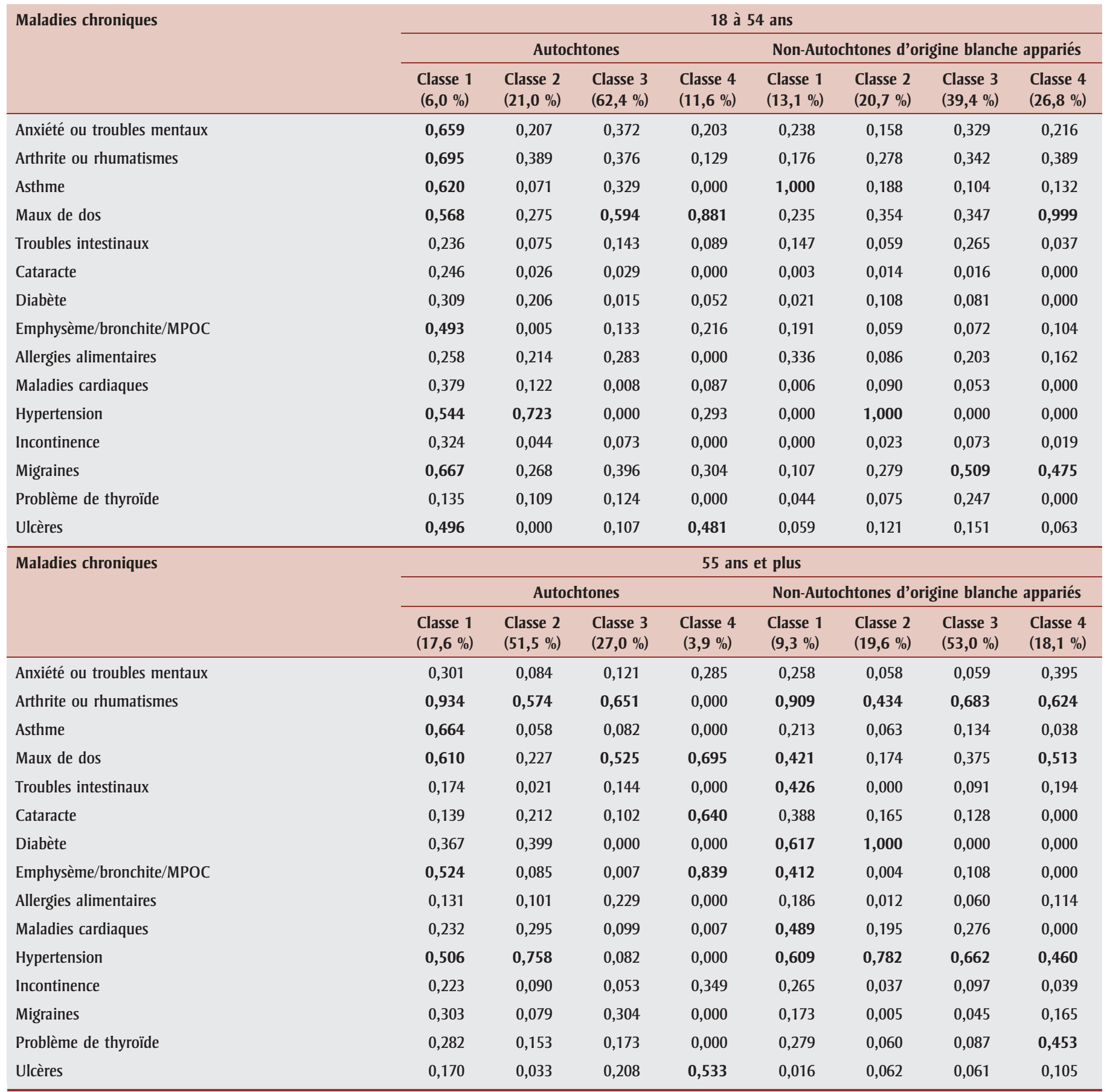

Abréviations : ESCC, Enquête sur la santé dans les collectivités canadiennes; MPOC, maladie pulmonaire obstructive chronique.

Remarque : Les valeurs en gras indiquent les maladies pour lesquelles la probabilité de réponse était de 0,400 ou plus.

constituée de cas d'arthrite ou de rhumatismes, de maux de dos, de troubles intestinaux, de diabète, de maladies cardiaques, d'hypertension et d'emphysème ou de bronchite ou de MPOC. Dans l'étude australienne ${ }^{22}$, les chercheurs ont observé une grappe équivalente de maladies (maladies cardiovasculaires, diabète, fatigue, hypertension, hypercholestérolémie, arthrite).

\section{Limites}

Les membres des Premières Nations qui vivent dans une réserve représentent une proportion importante de la population totale des Autochtones, mais ils étaient exclus de la présente étude; par conséquent, nos résultats sont uniquement représentatifs des Autochtones canadiens qui ne vivent pas dans une réserve. Les données ayant été tirées d'une enquête 
transversale, elles nous fournissent un instantané de la multimorbidité à un moment précis. Les maladies qui sont associées à des grappes peuvent donc changer au fil du temps. Bien que nous ayons apparié les répondants autochtones et les répondants non autochtones d'origine blanche en fonction de deux variables importantes (l'âge et le sexe), des différences peuvent subsister entre ces deux populations sur le plan des caractéristiques socioéconomiques, qui sont elles aussi susceptibles d'être associées à la multimorbidité. Un effet de confusion résiduel pourrait ainsi expliquer certaines des différences observées entre ces deux populations en ce qui concerne les grappes de maladies. Il pourrait également y avoir des différences en ce qui concerne l'accès aux services de santé entre les Autochtones et les non-Autochtones, ce qui pourrait entraîner une sous-déclaration des diagnostics de maladies chroniques. Cette sousdéclaration est plus susceptible d'affecter la population autochtone.

Par ailleurs, les enquêtes sont sujettes de manière générale à un biais d'autodéclaration, car il est parfois difficile de se souvenir avec exactitude des diagnostics que l'on pourrait avoir reçus et car certaines maladies sont considérées comme socialement indésirables.

Des travaux ultérieurs pourraient être menés pour déterminer dans quelle mesure les personnes présentant des grappes de maladies chroniques différentes ont des profils différents en ce qui concerne l'utilisation des services de santé, en particulier les soins d'urgence, les soins aigus, les soins primaires et les soins de soutien. Ce type de résultats nous permettrait de déterminer l'intérêt clinique et les répercussions financières de divers profils de multimorbidité.

\section{Conclusion}

Nos travaux mettent en lumière la dynamique des maladies chroniques concomitantes qui touchent les Autochtones et les non-Autochtones d'origine blanche au Canada. La prévalence de la multimorbidité était plus élevée chez la population d'Autochtones à l'étude que chez la population de non-Autochtones d'origine blanche appariée en fonction de l'âge et du sexe. Bien que l'on ait observé certaines similitudes en ce qui concerne les maladies qui avaient tendance à être concomitantes chez les groupes plus âgées d'Autochtones et de non-Autochtones d'origine blanche, il y avait certaines différences entre les groupes plus jeunes. La compréhension des différences quant aux maladies qui sont susceptibles d'être concomitantes chez des populations différentes peut favoriser l'élaboration de stratégies adaptées de prévention et de gestion.

Nous avons par ailleurs décelé un petit groupe de répondants autochtones dans le groupe des plus jeunes qui présente un profil complexe de maladies chroniques concomitantes. Les membres de ce groupe tireraient particulièrement profit des programmes de prévention et de gestion des maladies.

\section{Contributeurs}

JPK, LML et SS ont contribué à la conception et à l'élaboration de l'étude, de même qu'à l'acquisition, à l'analyse et à l'interprétation des données, et ont donné leur aval à la publication du présent article. JPK et LML étaient plus spécifiquement responsables de la rédaction de l'article et de la révision critique sur le plan du contenu intellectuel.

\section{Remerciements}

Les auteurs tiennent à remercier les employés du Centre de données de recherche de Statistique Canada à Winnipeg (Manitoba), et en particulier Ian Clara, pour leur aide sur le plan technique.

Bien que la présente étude ait été approuvée par Statistique Canada dans le cadre des exigences relatives à l'accès aux données, l'interprétation et les conclusions qui y sont présentées ne reflètent pas nécessairement celles de Statistique Canada.

JPK a reçu une bourse du Western Regional Training Centre for Health Services Research (WRTC) de l'Université du Manitoba et du Manitoba Health Research Council. LML est titulaire d'une chaire de recherche du Manitoba Health Research Council.

Il n’y avait aucun conflit d'intérêt.

\section{Références}

1. Van den Akker M, Buntinx F, Metsemakers JF, Roos S, Knottnerus JA. Multimorbidity in general practice: prevalence, incidence, and determinants of co-occurring chronic and recurrent diseases. J Clin Epidemiol. 1998;51:367-375.

2. McGann PE. Comorbidity in heart failure in the elderly. Clin Geriatr Med. 2001;16: 63148.

3. Patrick L, Knoefel F, Gaskowski P, Rexroth D. Medical comorbidity and rehabilitation efficiency in geriatric inpatients. J Am Geriatr Soc. 2001;49:1471-1477.

4. Wolff JL, Starfield B, Anderson G. Prevalence, expenditure, and complications of multiple chronic conditions in elderly. Arch Inter Med. 2002;162:2269-2276.

5. Orueta JF, Nuno-Solinis R, Garcia-Alvarez A, Alonso-Moran E. Prevalence of multimorbidity according to the deprivation level among the elderly in the Basque country. BMC Public Health. 2013;13:918.

6. Tucker-Seeley RD, Li Y, Sorensen G, Subramanian SV. Lifecourse socioeconomic circumstances and multimorbidity among older adults. BMC Public Health. 2011;11: 313.

7. Mathur R, Hull SA, Badrick E, Robson J. Cardiovascular multimorbidity : the effect of ethnicity on prevalence and risk factor management. Br J Gen Pract. 2011;61:e262e270.

8. Shadmi E. Multimorbidity and equity in health. Int J Equity Health. 2013;12:59.

9. Schafer I, von Leitner EC, Schon G, et al. Multimorbidity patterns in the elderly: a new approach of disease clustering identifies complex interrelations between chronic conditions. Plos ONE. 2010;5(12):e15941.

10. Prados-Torres A, Poblador-Plou B, CalderonLarranaga A et collab. Multimorbidity patterns in primary care: Interactions among chronic diseases using factor analysis. Plos ONE. 2012;7(2):e32190. 
11. Cornell JE, Pugh JA, Williams JW et collab. Multimorbidity clusters: Clustering binary data from a large administrative medical database. Appl Multivar Res. 2008;12:163182.

12. Marengoni A, Rizzuto D, Wang HX, Winblad B, Fratiglioni L. Patterns of chronic multimorbidity in the elderly population. J Am Geriatr Soc. 2009;57:225-230.

13. Newcomer SR, Steiner JF, Bayliss EA. Identifying subgroups of complex patients with cluster analysis. Am J Manag Care. 2011;17(8):e324-e332.

14. Kaldor J, Clayton D. Latent class analysis in chronic disease epidemiology. Stat Med. 1985;4(3):327-335.

15. Magidson J, Vermunt JK. Latent class models for clustering: a comparison with K-means. Can J Market Res. 2002;20:37-44.

16. Deering KN, Lix LM, Bruce S, Young TK. Chronic diseases and risk factors in Canada's northern populations: longitudinal and geographic comparisons. Can J Public Health. 2009;100(1):14-17.

17. Agence de la santé publique du Canada. Le diabète au Canada : perspective de santé publique sur les faits et chiffres [Internet]. Ottawa (Ont.) : Agence de la santé publique du Canada; 2011 [consultation le 11 janv. 2014]. Consultable en ligne à la page : http:// www.phac-aspc.gc.ca/cd-mc/publications /diabetes-diabete/facts-figures-faits-chiffres -2011/index-fra.php

18. Statistique Canada. Enquête sur la santé dans les collectivités canadiennes. Information détaillée pour 2005 (cycle 3.1) [Internet]. Ottawa (Ont.) : Statistique Canada; 2005 [consultation le 30 déc. 2013]. Consultable en ligne à la page : http://www23.statcan .gc.ca/imdb/p2SV_f.pl?Function = getSurvey \&SurvId $=1630 \&$ InstaId $=22642 \&$ SDDS $=3226$

19. Statistique Canada. Enquête sur la santé dans les collectivités canadiennes, Cycle 3.1. Questionnaire final [Internet]. Ottawa (Ont.) : Statistique Canada; 2005 [consultation le 30 déc. 2013]. PDF (1,4 Mo) téléchargeable à partir du lien : http://www.23. statcan.gc.ca/imdb-bmdi/instrument/3226_ Q1_V3-fra.pdf

20. Stuart EA. Matching methods for causal inference: a review and a look forward. Stat Sci. 2010;25(1):1-21.
21. Lanza ST, Collins LM. A new SAS procedure for latent transition analysis: Transitions in dating and sexual risk behaviour. Dev Psychol. 2008;44(2):446-456.

22. Holden L, Scuffham PA, Hilton MF, Muspratt A, Ng SK, Whiteford HA. Patterns of multimorbidity in working Australians. Popul Health Metrics. 2011;9:15.

23. Akaike H. A new look at the statistical model identification. IEEE Trans Autom. 1974;19:716-723.

24. Schwartz G. Estimating the dimension of a model. Ann Statist. 1978; 6:461-464.

25. Lanza ST, Collins LM, Lemmon DR et collab. Proc LCA: a SAS procedure for latent class analysis. Struct Equ Modeling. 2007;14(4):671-694.

26. PROC LCA \& PROC LTA (Version 1.2.7) [logiciel]. Penn State: The Methodology Center, PA; 2011 [consultation le 20 juill. 2013]. Consultable en ligne à partir de la page : http://methodology.psu.edu

27. SAS Institute Inc. SAS/STAT user's guide. Cary (NC) : SAS Institute Inc; 2004. 